\title{
HIGH FREQUENCY VIBRATIONS OF CRYSTAL PLATES*
}

\author{
BY \\ R. D. MINDLIN \\ Columbia University
}

1. Introduction. In this paper, Cauchy's [1] two-dimensional equations of coupled flexural and extensional motion of crystal plates are extended to the next higher order of approximation so as to accommodate the frequencies of the two lowest thicknessshear modes. The equations obtained are also extensions of previous equations [2] in which flexure and the same thickness-shear modes were included, but coupling with extensional modes was omitted. The new equations are deduced from the three-dimensional equations of linear elasticity by a procedure based on the series expansion methods of Cauchy [1] and Poisson [3] and the variational method of Kirchhoff [4]. Comparison of the appropriate solution of the resulting equations with Ekstein's [5] solution of the three-dimensional equations, for an infinite plate, reveals close agreement between the two frequency spectra, over the extended range of frequencies, for all five branches of the spectrum of the two-dimensional equations. This indicates that solutions of these equations, for bounded plates, will give reliable results, over the extended range of frequencies, since the modes of bounded plates, in that range, are composed essentially of coupled overtones of the first five modes of vibration of an infinite plate.

In addition to the derivation of the approximate equations, theorems of uniqueness and orthogonality are established and some general conclusions are drawn regarding solutions in rectangular coordinates and vibrations of rectangular plates.

2. Expansion in power series. The plate is referred to rectangular coordinates $x_{i}(i=1,2,3)$ with $x_{1}$ and $x_{3}$ in the middle plane and the faces at $x_{2}= \pm h$. The components of displacement $u_{i}(j=1,2,3)$ are expanded in power series of the thicknesscoordinate:

$$
u_{i}=\sum_{n=0}^{\infty} x_{2}^{n} u_{i}^{(n)}=u_{i}^{(0)}+x_{2} u_{i}^{(1)}+x_{2}^{2} u_{i}^{(2)} \cdots,
$$

where the $u_{i}^{(n)}$ are functions of $x_{1}, x_{3}$ and the time, $t$, only.

Stress-equations of motion. The series expression for $u_{i}$ is substituted in

$$
\int_{V}\left(T_{i i, i}-\rho u_{i, t}\right) \delta u_{i} d V=0
$$

which is obtained from the variational equation of motion, deduced from Hamilton's principle [6]. In (2), the integration is over the volume, $V$, of the plate; the $T_{i i}$ are the components of stress; $\rho$ is the density; and the summation convention for repeated indices is employed, as is the comma notation for differentiation with respect to the $x_{i}$ and the time $t$.

When the integration with respect to $x_{2}$, from $-h$ to $h$, is performed in (2), the result is

${ }^{*}$ Received September 26, 1960. 


$$
\int_{\Lambda} \sum_{n=0}^{\infty}\left(T_{i j, i}^{(n)}-n T_{2 i}^{(n-1)}+F_{i}^{(n)}-\rho \sum_{m=0}^{\infty} H_{m n} u_{j, t i}^{(m)}\right) \delta u_{i}^{(n)} d A=0,
$$

where $A$ is the area of the plate and

$$
\begin{gathered}
T_{i i}^{(n)} \equiv \int_{-h}^{h} x_{2}^{n} T_{i i} d x_{2}, \quad F_{i}^{(n)} \equiv\left[x_{2}^{n} T_{2 i}\right]_{-h}^{h} \\
H_{m n} \equiv \begin{cases}2 h^{m+n+1} /(m+n+1), & m+n \\
0, & m+n \text { odd }\end{cases}
\end{gathered}
$$

Since (3) must hold for all $A$ and arbitrary $\delta u_{i}^{(n)}$, the quantity in parentheses must. vanish and we arrive at the stress-equations of motion of order $n$

$$
T_{i j, i}^{(n)}-n T_{2 i}^{(n-1)}+F_{i}^{(n)}=\rho \sum_{m=0}^{\infty} H_{m n}^{(m)} u_{i, t t} .
$$

Strain. The three-dimensional components of strain, $S_{i i}$, are expressed in terms of the $u_{i}$ by

$$
S_{i j}=\frac{1}{2}\left(u_{i, j}+u_{i, i}\right) .
$$

Upon substituting (1) in (5) we obtain, after a rearrangement of terms,

$$
S_{i j}=\sum_{n=0}^{\infty} x_{2}^{n} S_{i i}^{(n)}
$$

where

$$
S_{i j}^{(n)} \equiv \frac{1}{2}\left[u_{i, i}^{(n)}+u_{i, i}^{(n)}+(n+1)\left(\delta_{2 i} u_{i}^{(n+1)}+\delta_{i 2} u_{i}^{(n+1)}\right)\right]
$$

in which $\delta_{i j}$ is the Kronecker symbol.

Stress-strain relations. In three dimensions,

$$
\begin{array}{ll}
T_{i j}=c_{i j k l} S_{k l}, & c_{i j k l}=c_{i i k l}=c_{k l i i}, \\
S_{i j}=s_{i j k l} T_{k l}, & s_{i j k l}=s_{i i k l}=s_{k l i j},
\end{array}
$$

where $c_{i j k l}$ and $s_{i j k l}$ are the elastic stiffnesses and compliances, respectively.

The expressions for the two-dimensional $T_{i j}^{(n)}$ in terms of the $S_{i j}^{(n)}$ are obtained by inserting (6) in (7) and (7) in the first of (4), with the result

$$
T_{i j}^{(n)}=c_{i i k l} \sum_{m=0}^{\infty} H_{m n} S_{k l}^{(m)} .
$$

Energy-densities. A strain-energy-density, $U$, and a kinetic energy-density, $K$, both reckoned per unit area of the plate, are defined by

$$
\begin{gathered}
U \equiv \frac{1}{2} \int_{-h}^{h} c_{i j k l} S_{i j} S_{k l} d x_{2}=\frac{1}{2} c_{i j k l} \sum_{m=0}^{\infty} \sum_{n=0}^{\infty} H_{m n} S_{i j}^{(m)} S_{k l}^{(n)}, \\
K \equiv \frac{1}{2} \int_{-h}^{h} \rho u_{j, t} u_{i, t} d x_{2}=\frac{1}{2} \rho \sum_{m=0}^{\infty} \sum_{n=0}^{\infty} H_{m n} u_{i, t}^{(m)} u_{j, \ell}^{(n)} .
\end{gathered}
$$

It may be noted that

$$
T_{i j}^{(n)}=\partial U / \partial S_{i i}^{(n)} ; \quad \partial S_{i j}^{(n)} / \partial S_{i i}^{(n)}=0, \quad i \neq j .
$$


3. Truncation of series. We retain only the zero and first order components of stress and strain and we write, tentatively, from (9),

$$
\begin{aligned}
& T_{i j}^{(0)}=2 h c_{i j k l} S_{k l}^{(0)}, \\
& T_{i j}^{(1)}=\frac{2}{3} h^{3} c_{i j k l} S_{k l}^{(1)} .
\end{aligned}
$$

The components $S_{i j}^{(0)}$ and $S_{i j}^{(1)}$ involve only the displacements $u_{i}^{(0)}, u_{i}^{(1)}$ and $u_{i}^{(2)}$ and we neglect those of higher order. Then, following Cauchy, we neglect the velocity $u_{2, t}^{(1)}$ in the kinetic energy and equations of motion and provide for the free development of the strain $S_{22}^{(0)}\left(=u_{2}^{(1)}\right)$ by setting $T_{22}^{(0)}=0$ in (10). The condition $T_{22}^{(0)}=0$ permits the elimination of $S_{22}^{(0)}$ from (10), with the result, again tentative,

$$
T_{i j}^{(0)}=2 h g_{i j k l} S_{k l}^{(0)},
$$

where

$$
g_{i j k l}=c_{i j k l}-c_{i j 22} c_{22 k l} / c_{2222} .
$$

It may be observed that, because of the form of $g$, only five components of stress and five components of strain remain in (12).

The first order terms, $T_{i i}^{(1)}$ and $S_{i j}^{(1)}$, are treated in a similar manner except that all three velocities $u_{j, t}^{(2)}$ are neglected and free development of the three strains $S_{2 j}^{(1)}$ is accommodated by setting $T_{2 i}^{(1)}=0$ in (11). Upon elimination of the $S_{2 i}^{(1)}$ from (11), we arrive at

$$
T_{a b}^{(1)}=\frac{2}{3} h^{3} \gamma_{a b c d} S_{c d}^{(1)} ; \quad a, b, c, d=1,3,
$$

where

$$
\gamma_{a b c d}=A_{a b c d} /\left|s_{a b c d}\right|
$$

in which $\left|s_{a b c d}\right|$ is the determinant

$$
\left|s_{a b c d}\right|=\left|\begin{array}{rrr}
s_{1111} & s_{1133} & 2 s_{1113} \\
s_{3311} & s_{3333} & 2 s_{3313} \\
2 s_{1311} & 2 s_{1333} & 4 s_{1313}
\end{array}\right|
$$

and $A_{a b c d}$ is the cofactor of that element of $\left|s_{a b c d}\right|$ which contains $s_{a b c d}$.

As the final step in the process of truncation, the thickness-shear strains $S_{21}^{(0)}$ and $S_{23}^{(0)}$ are replaced by $k_{1} S_{21}^{(0)}$ and $k_{3} S_{23}^{(0)}$ in the strain-energy-density, where $k_{1}$ and $k_{3}$ are correction-factors which may be used to adjust the thickness-shear frequencies to their exact values; thus compensating, in part, for the omission of terms of higher order in the series expansions. The correction-factors may be introduced conveniently by replacing $g_{i j k l}$ with

$$
g_{i j k l}^{*} \equiv k_{i+i-2}^{m} k_{k+l-2}^{n} g_{i j k l} \quad \text { (no sum), }
$$

where $m$ and $n$ are the powers

$$
m=\cos ^{2}(i j \pi / 2), \quad n=\cos ^{2}(k l \pi / 2) .
$$

Thus $k_{i+i-2}^{m}$ (or $k_{k+l-2}^{n}$ ) is equal to $k_{1}, k_{3}$ or 1 according as $i+j$ (or $k+l$ ) in $g_{i i k l}$ is 3,5 or neither, respectively. 
4. Recapitulation. The equations remaining, after truncation of the series and adjustment of the terms retained, are [7]

Energy-densities*

$$
\begin{aligned}
& U=h g_{i j k l}^{*} S_{i j}^{(0)} S_{k l}^{(0)}+\frac{1}{3} h^{3} \gamma_{a b c d} S_{a b}^{(1)} S_{c d}^{(1)}, \\
& K=\rho h\left(u_{i, t}^{(0)} u_{i, t}^{(0)}+\frac{1}{3} h^{2} u_{a, t}^{(1)} u_{a, t}^{(1)}\right) .
\end{aligned}
$$

Stress-strain relations

$$
\begin{aligned}
& T_{i j}^{(0)}=\partial U / \partial S_{i j}^{(0)}=2 h g_{i j k l}^{*} S_{k l}^{(0)} \\
& T_{a b}^{(1)}=\partial U / \partial S_{a b}^{(1)}=\frac{2}{3} h^{3} \gamma_{a b c d} S_{c d}^{(1)},
\end{aligned}
$$

where $\partial S_{i j}^{(0)} / \partial S_{i i}^{(0)}=0, i \neq j ; \partial S_{a b}^{(1)} / \partial S_{b a}^{(1)}=0, a \neq b$.

Stress-equations of motion

$$
\begin{aligned}
T_{i j, i}^{(0)}+F_{j}^{(0)} & =2 h \rho u_{j, t t}^{(0)}, \\
T_{a b, a}^{(1)}-T_{2 b}^{(0)}+F_{b}^{(1)} & =\frac{2}{3} h^{3} \rho u_{b, t \ell}^{(1)} .
\end{aligned}
$$

Strain-displacement relations

$$
\begin{gathered}
S_{i j}^{(0)}=\frac{1}{2}\left(u_{i, j}^{(0)}+u_{i, i}^{(0)}+\delta_{2 j} u_{i}^{(1)}+\delta_{2 i} u_{i}^{(1)}\right), \\
S_{a b}^{(1)}=\frac{1}{2}\left(u_{a, b}^{(1)}+u_{b, a}^{(1)}\right) .
\end{gathered}
$$

Note that, although $S_{22}^{(0)}=u_{2}^{(1)}$ is contained in (19), neither of the two appears in any other of the Eqs. (13)-(20) because the zero order components of strain always occur as products with $g_{i j k l}^{*}$ and the latter is zero when $i j$ or $k l$ is 22 . Hence, there are only eight components of strain and eight components of stress to be considered. The components of strain are related through the four compatibility equations

$$
\begin{array}{ll}
S_{11,33}^{(0)}+S_{33,11}^{(0)}=2 S_{13,13}^{(0)}, & S_{33,1}^{(1)}-S_{13,3}^{(1)}=S_{23,13}^{(0)}-S_{12,33}^{(0)}, \\
S_{11,33}^{(1)}+S_{33,11}^{(1)}=2 S_{13,13}^{(1)}, & S_{11,3}^{(1)}-S_{31,1}^{(1)}=S_{21,31}^{(0)}-S_{32,11}^{(0)} .
\end{array}
$$

Displacement-equations of motion

$$
\begin{gathered}
2 h g_{i j k l}^{*}\left(u_{k, l i}^{(0)}+\delta_{2 k} u_{l, i}^{(1)}\right)+F_{i}^{(0)}=2 h \rho u_{j, t t}^{(0)}, \\
\frac{2}{3} h^{3} \gamma_{a b c d} u_{c, d a}^{(1)}-2 h g_{2 b k l}^{*}\left(u_{k, l}^{(0)}+\delta_{2 k} u_{l}^{(1)}\right)+F_{b}^{(1)}=\frac{2}{3} h^{3} \rho u_{b, t \ell}^{(1)} .
\end{gathered}
$$

These equations are closely related to several predecessors. Thus, if the thickness-shear and flexure are eliminated by setting the transverse shear forces $T_{2 a}^{(0)}$ and the couples $T_{a b}^{(1)}$ and $F_{b}^{(1)}$ equal to zero, the first and third of (22) reduce to the Cauchy-Voigt [1, 8] equations of low frequency extensional motion of thin plates:

$$
2 h \gamma_{a b c d} u_{c, a d}^{(0)}+F_{b}^{(0)}=2 h \rho u_{b, t t}^{(0)} .
$$

Conversely, if the extensional deformation is suppressed by setting $u_{a}^{(0)}=F_{a}^{(0)}=0$, (22) and (23) become equations of coupled thickness-shear and flexural vibrations [2]

$$
\begin{gathered}
2 h g_{a 2 b 2}^{*}\left(u_{2, b a}^{(0)}+u_{b, a}^{(1)}\right)+F_{2}^{(0)}=2 h \rho u_{2, t \ell}^{(0)} \\
\frac{2}{3} h^{3} \gamma_{a b c d} u_{c, d a}^{(1)}-2 h g_{a 2 b 2}^{*}\left(u_{2, a}^{(0)}+u_{a}^{(1)}\right)+F_{b}^{(1)}=\frac{2}{3} h^{3} \rho u_{b, t \ell}^{(1)} .
\end{gathered}
$$

${ }^{*}$ Here and in the sequel: indices $i, j, k, l$ range over $1,2,3$; indices $a, b, c, d$ range over $1,3$. 
Alternatively, in the case of isotropy, (22) and (23) become

$$
\begin{gathered}
\mu u_{b, a a}^{(0)}+\left(\lambda^{\prime}+\mu\right) u_{a, a b}^{(0)}+\frac{1}{2} h^{-1} F_{b}^{(0)}=\rho u_{b, t t}^{(0)}, \\
k^{2} \mu\left(u_{2, a a}^{(0)}+u_{a, a}^{(1)}\right)+\frac{1}{2} h^{-1} F_{2}^{(0)}=\rho u_{2, t t}^{(0)}, \\
\mu u_{b, a a}^{(1)}+\left(\lambda^{\prime}+\mu\right) u_{a, a b}^{(1)}-3 h^{-2} k^{2} \mu\left(u_{2, b}^{(0)}+u_{b}^{(1)}\right)+\frac{3}{2} h^{-3} F_{b}^{(1)}=\rho u_{b, t l}^{(1)},
\end{gathered}
$$

where $\lambda^{\prime}=2 \mu \lambda /(\lambda+2 \mu)$ and $\lambda$ and $\mu$ are Lamés constants. Equations (27)-(29) are also the isotropic forms of (24)-(26). Equations (27) are Poisson's equations of low frequency extensional motion of thin plates [ 3 and 6, p. 497] or, what is the same, the equations of motion in generalized plane stress. Equations (28) and (29) are equations of flexural motion of isotropic plates with rotatory inertia and transverse shear deformation taken into account [9]. In the one-dimensional case they have the same form as the Timoshenko beam-equations [10] and in the case of equilibrium they have the same form as Reissner's plate-equations [11]. Finally, by setting the transverse shear deformation, $S_{2 a}^{(0)}$, and the rotatory inertia, $\frac{2}{3} h^{3} \rho u_{a, t t}^{(1)}$, equal to zero, (22) and (23) may be reduced to equations equivalent to Cauchy's [1].

5. Correction factors. The values of $k_{1}$ and $k_{3}$ are found by equating the thicknessshear frequencies obtained from (23) with the corresponding ones obtained from the three-dimensional equations.

In (23), let $F_{b}^{(1)}=u_{i}^{(0)}=0$ and $u^{(1)}=A_{b}^{(1)} \exp i w t$, where the $A_{b}^{(1)}$ are constants. Then (23) become

$$
g_{2 b 2 d}^{*} A_{d}^{(1)}=\frac{1}{3} \rho h^{2} \omega^{2} A_{b}^{(1)} .
$$

Upon equating to zero the determinant of the coefficients of the $A_{b}^{(1)}$, we have

$$
\left|g_{2 b 2 d}^{*}-g \delta_{b d}\right|=0, \quad g=\frac{1}{3} \rho h^{2} \omega^{2}
$$

which gives two frequencies, say $\omega_{1}$ and $\omega_{3}$. These are to be equated to the two lowest roots of the frequency equation

$$
\left|c_{2 i 2 l}-c \delta_{i l}\right|=0, \quad c=4 \rho h^{2} \omega^{2} / \pi^{2}
$$

for the fundamental thickness-modes, as obtained from the three-dimensional equations [12]. The two equations yield a pair of equations which may be solved for $k_{1}$ and $k_{3}$ in terms of ratios of the $c_{i j k l}$.

As an example, consider the case of monoclinic symmetry with $x_{1}$ the digonal axis so that $c_{2122}$ and $c_{2123}$ are zero. Then the roots of (30) are

$$
\begin{array}{ll}
g_{1}=k_{1}^{2} g_{2121}=k_{1}^{2} c_{2121}, & \omega_{1}^{2}=3 g_{1} / \rho h^{2}, \\
g_{3}=k_{3}^{2} g_{2323}=k_{3}^{2}\left(c_{2323}-c_{2223}^{2} / c_{2222}\right), & \omega_{3}^{2}=3 g_{3} / \rho h^{2}
\end{array}
$$

and the two lowest roots of (31) are

$$
\begin{array}{ll}
c_{1}=c_{2121}, & \omega_{1}^{2}=\pi^{2} c_{1} / 4 \rho h^{2}, \\
c_{3}=\frac{1}{2}\left\{c_{2222}+c_{2323}-\left[\left(c_{2222}-c_{2323}\right)^{2}+4 c_{2223}^{2}\right]^{1 / 2}\right\}, & \omega_{3}^{2}=\pi^{2} c_{3} / 4 \rho h^{2} .
\end{array}
$$

Equating corresponding frequencies, we find

$$
k_{1}^{2}=\pi^{2} / 12, \quad k_{3}^{2}=\pi^{2} c_{3} / 12 g_{2323} .
$$


6. Straight-crested waves in a monoclinic plate. The adequacy of Eqs. (13)-(23), for the prediction of frequencies of vibration of bounded crystal plates, may be judged by a comparison of the five-branched frequency spectrum of an infinite plate, as obtained from (22) and (23), with the first five branches of the spectrum obtained from the threedimensional equations. This is because the modes of vibration of a bounded plate are composed essentially of the anharmonic overtones of the modes of an infinite plate, as described in Secs. 16 and 17 of Ref [13]. Because of its importance in technology, the frequency spectrum of straight-crested waves traveling along the digonal axis in the AT cut of quartz is chosen as the basis of comparison.

Quartz is a trigonal crystal (six elastic constants) and the AT cut is a plate which contains a digonal axis and whose normal makes an angle of $35^{\circ} 15^{\prime}$ with the trigonal axis [14]. When referred to rectangular axes in and normal to the plane of such a plate, the stress-strain relation has monoclinic symmetry (thirteen constants). With $x_{1}$ the digonal axis in the plane of the plate and $x_{2}$ the axis normal to the plate, the values of the thirteen constants, as computed from Bechmann's [15] values of the six principal constants, are, in units of $10^{9}$ newtons per square meter,

$$
\begin{aligned}
& c_{1111}=86.74 \quad c_{2323}=38.61 \quad c_{1122}=-8.26 \quad c_{2233}=-7.42 \\
& c_{2222}=129.77 \quad c_{3131}=68.81 \quad c_{1133}=27.15 \quad c_{2223}=5.70 \\
& c_{3333}=102.84 \quad c_{1212}=29.01 \quad c_{1123}=-3.65 \quad c_{3323}=9.92 \\
& c_{1213}=2.53
\end{aligned}
$$

while

$$
c_{1113}=c_{2213}=c_{3313}=c_{2313}=c_{1112}=c_{2212}=c_{3312}=c_{2312}=0 .
$$

Then, in (22) and (23), let $F_{i}^{(0)}=F_{b}^{(1)}=0$ and

$$
u_{i}^{(0)}=A_{i}^{(0)} \exp i\left(\xi x_{1}-\omega t\right), \quad u_{b}^{(1)}=A_{b}^{(1)} \exp i\left(\xi x_{1}-\omega t\right) .
$$

As a result, it is found that the first of (22) is coupled only with the second of (23) and the first (23) is coupled only with the second and third of (22). Thus, the general quintic, relating $\omega^{2}$ and $\xi^{2}$, reduces, in this case, to a cubic and a quadratic

$$
\begin{gathered}
\left|\begin{array}{ccc}
g_{1212}^{*} \xi^{2}-\rho \omega^{2} & g_{1312}^{*} \xi^{2} & -i g_{1212}^{*} \xi \\
g_{1312}^{*} \xi^{2} & c_{1313} \xi^{2}-\rho \omega^{2} & -i g_{1312}^{*} \xi \\
3 i h^{-2} g_{1212}^{*} \xi & 3 h^{-2} g_{1312} \xi & \gamma_{111} \xi^{2}+3 h^{-2} g_{1212}^{*}-\rho \omega^{2}
\end{array}\right|=0 \\
\left|\begin{array}{cc}
g_{1111} \xi^{2}-\rho \omega^{2} & -i g_{1123}^{*} \xi \\
3 i h^{-2} g_{1123}^{*} \xi & \gamma_{1313} \xi^{2}+3 h^{-2} g_{2323}^{*}-\rho \omega^{2}
\end{array}\right|=0 .
\end{gathered}
$$

The roots of these equations are plotted in Fig. 1 to dimensionless coordinates

$$
\Omega=\omega /(\pi / 2 h)\left(c_{1212} / \rho\right)^{1 / 2}, \quad \phi=2 \xi h / \pi
$$

The three branches marked flexure, face-shear and thickness-shear are the roots of the cubic, while the branches marked extension and thickness-twist are the roots of the quadratic.

The five branches are to be compared with the first five branches of the analogous solution, by Ekstein [5], of the three-dimensional equations. The results of detailed 
computations $[16,17]$ show correspondence to at least three significant figures over most of the range covered by Fig. 1. The asymptotic behaviors of the four branches which intersect at $\Omega=0, \phi=0$

$$
\begin{aligned}
& \Omega=\phi^{2}(\pi / 2)\left(\gamma_{1111} / 3 c_{1212}\right)^{1 / 2} \quad \text { (flexure), } \\
& \Omega=\phi\left(\gamma_{1313} / c_{1212}\right)^{1 / 2} \quad \text { (face-shear), } \\
& \Omega=\phi\left(\gamma_{1111} / c_{1212}\right)^{1 / 2} \quad \text { (extension), } \\
& \Omega=-\phi^{2}(\pi / 2)\left(\gamma_{1111} / 3 c_{1212}\right)^{1 / 2} \quad \text { (thickness-shear) }
\end{aligned}
$$

are the same, for the approximate and exact equations, due to the method adopted in the derivation of the former. The remaining branch comes to zero frequency at

$$
i_{\phi}=\left(\gamma_{1111} c_{3} / \gamma_{1313} g_{1111}\right)^{1 / 2}=0.7456
$$

in the approximate equations and $i \phi=0.7467$ in the exact equations. In the high frequency range the important frequencies, in the usual applications, are near $\Omega=1$ and the important branches are the thickness-shear and flexural branches. At $\Omega=1$, the thickness-shear branch is exact, due to the choice $k_{1}^{2}=\pi^{2} / 12$; the flexural branch in the approximate equations has $\phi=1.2483$ whereas the exact value is $\phi=1.2417$.

The approximate equations should not be used for frequencies so high that branches higher than the fifth cannot be neglected. In the AT cut of quartz the sixth branch has a real minimum at a value of $\Omega$ slightly less than 2.0 [16]; however, due to the likelihood

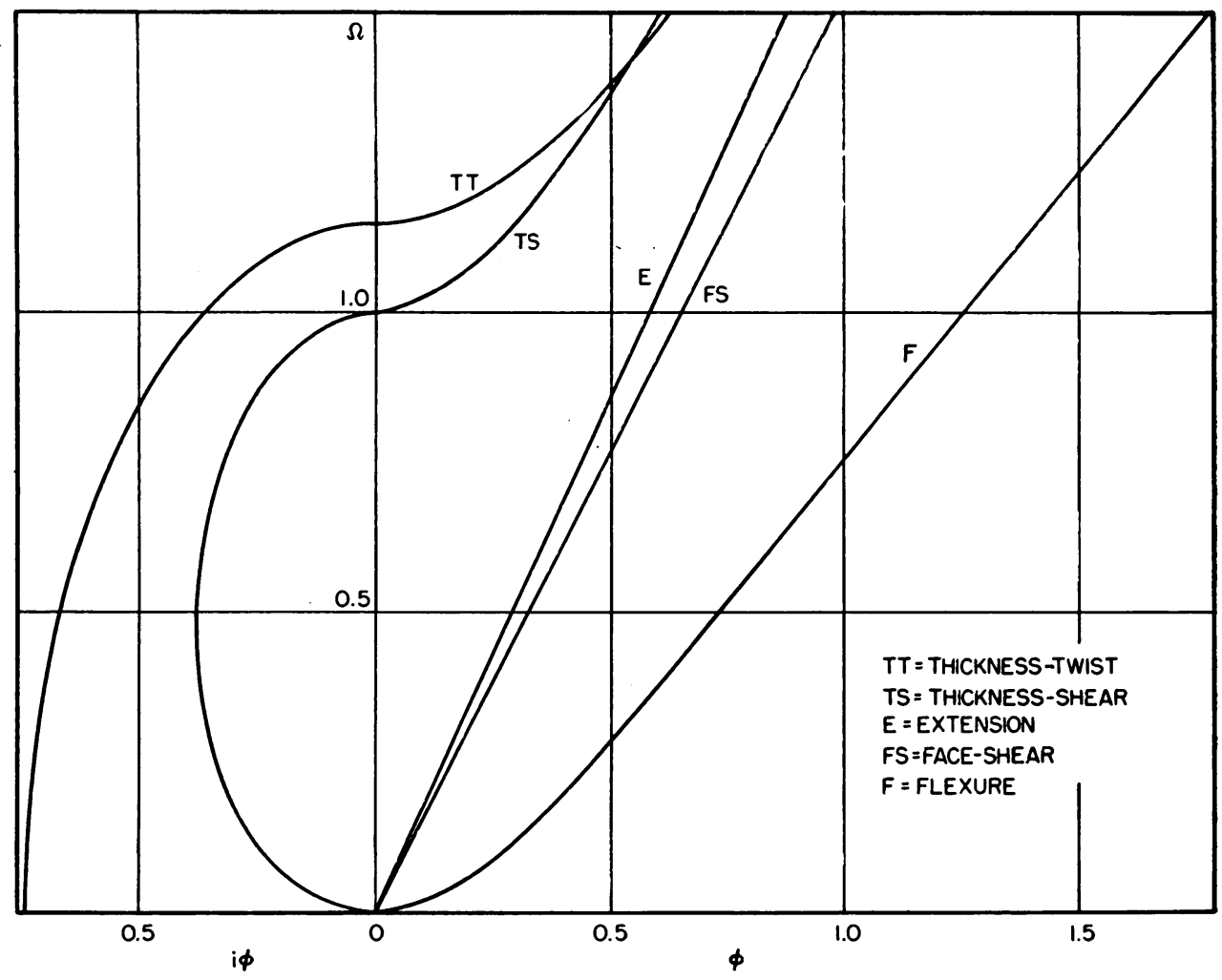

Fig. 1. Frequency spectrum. 
of the contribution of an edge-mode from the complex segments of the sixth and seventh branches $[17,18]$, applications of the equations should be confined to $\Omega<1.5$.

7. Uniqueness of solution. A theorem analogous to Neumann's* leads to initial and boundary conditions sufficient to assure a unique solution of equations (15)-(20).

Consider two systems of the five displacements, eight strains (omitting $S_{22}^{(0)}$ ) and eight stresses which satisfy the eight stress-strain equations (15)-(16), the five stressequations of motion (17)-(18) and the eight strain-displacement equations (19)-(20). Let the differences between corresponding components of displacement, strain and stress constitute a "difference-system" of those quantities. We form the equation

$$
\begin{aligned}
\int_{t_{0}}^{t} d t \int_{A}\left[\left(T_{i i, i}^{(0)}+F_{i}^{(0)}-2 h \rho u_{i, t t}^{(0)}\right) u_{i, t}^{(0)}\right. & \\
& \left.+\left(T_{a b, a}^{(1)}-T_{2 b}^{(0)}+F_{b}^{(1)}-\frac{2}{3} h^{3} \rho u_{b, t t}^{(1)}\right) u_{b, t}^{(1)}\right] d A=0
\end{aligned}
$$

in which all the components are those of the difference-system and $t_{0}$ is an initial time. Now

$$
2 h \rho \int_{t_{0}}^{t} d t \int_{A}\left(u_{i, t}^{(0)} u_{i, t}^{(0)}+\frac{1}{3} h^{2} u_{b, t}^{(1)} u_{b, t}^{(1)}\right) d A=\int_{A}\left(K-K_{0}\right) d A,
$$

where $K$ is the kinetic energy-density of the difference-system and $K_{0}$ is its value at $t_{0}$. Also

$$
\begin{aligned}
T_{i, i, i}^{(0)} u_{i, t}^{(0)}+\left(T_{a b, a}^{(1)}-T_{2 b}^{(0)}\right) & u_{b, t}^{(1)} \\
= & \left(T_{i j}^{(0)} u_{j, t}^{(0)}\right)_{, i}+\left(T_{a b}^{(1)} u_{b, t}^{(1)}\right)_{, a}-T_{i i}^{(0)} u_{i, i t}^{(0)}-T_{a b}^{(1)} u_{b, a t}^{(1)}-T_{2 b}^{(0)} u_{b, t}^{(1)}, \\
= & \left(T_{a i}^{(0)} u_{i, t}^{(0)}+T_{a b}^{(1)} u_{b, t}^{(1)}\right)_{, a}-T_{i i}^{(0)}\left(u_{i, i}^{(0)}+\delta_{2 i} u_{i}^{(1)}\right)_{, t}-T_{a b}^{(1)} u_{b, a t}^{(1)}, \\
= & \left(T_{a i}^{(0)} u_{i, t}^{(0)}+T_{a b}^{(1)} u_{b, t}^{(1)}\right)_{, a}-\frac{1}{2} T_{i i}^{(0)}\left(u_{i, i}^{(0)}+u_{i, i}^{(0)}+\delta_{2 i} u_{i}^{(1)}\right. \\
& \left.+\delta_{2 i} u_{i}^{(1)}\right)_{, t}+\frac{1}{2} T_{a b}^{(1)}\left(u_{b, a}^{(1)}+u_{a, b}^{(1)}\right)_{, t}, \\
= & \left(T_{a j}^{(0)} u_{i, t}^{(0)}+T_{a b}^{(1)} u_{b, t}^{(1)}\right)_{, a}-\left(\partial U / \partial S_{i i}^{(0)}\right) S_{i, t}^{(0)}-\left(\partial U / \partial S_{a b}^{(1)}\right) S_{a b, t}^{(1)}, \\
= & \left(T_{a i}^{(0)} u_{i, t}^{(0)}+T_{a b}^{(1)} u_{b, t}^{(1)}\right)_{, a}-U_{, t},
\end{aligned}
$$

where $U$ is the strain-energy-density of the difference-system. Hence

$$
\begin{aligned}
\int_{t_{0}}^{t} d t \int_{A}\left[T_{i, i}^{(0)} u_{i, t}^{(0)}+\right. & \left.\left(T_{a b, a}^{(1)}-T_{2 b}^{(0)}\right) u_{b, t}^{(1)}\right] d A \\
= & \int_{t_{0}}^{t} d t \oint_{c} n_{a}\left(T_{a i}^{(0)} u_{i, t}^{(0)}+T_{a b}^{(1)} u_{b, t}^{(1)}\right) d s-\int_{A}\left(U-U_{0}\right) d A
\end{aligned}
$$

in which the line integral is around the boundary, $C$, of the plate and the $n_{a}$ are the direction cosines of the outward normal in the plane of the plate. In the transformation from the surface integral to the line integral, it is assumed that the compatibility equations (21) are satisfied. Finally, using (35) and (36) in (34),

$$
\begin{aligned}
\int_{A}(U+K) d A=\int_{A}\left(U_{0}+K_{0}\right) d A+ & \int_{t_{0}}^{t} d t \int_{A}\left(F_{i}^{(0)} u_{i, t}^{(0)}+F_{b}^{(1)} u_{b, t}^{(1)}\right) d A \\
& +\int_{t_{0}}^{t} d t \oint_{C} n_{a}\left(T_{a i}^{(0)} u_{i, t}^{(0)}+T_{a b}^{(1)} u_{b, t}^{(1)}\right) d s .
\end{aligned}
$$

${ }^{*}$ Reference [6], p. 176. 
Then conditions sufficient for a unique solution are established by the usual argument based on the vanishing of the right hand side of (37) and the positive definiteness of $U$ and $K$. It may be seen that, in addition to the initial values of $u_{i}^{(0)}, u_{a}^{(1)}, u_{j, t}^{(0)}$ and $u_{a, t}^{(1)}$, there are five conditions to be specified at each point of the interior of the plate and five conditions at each point of the edge. In terms of orthogonal coordinates $\alpha, \beta, x_{2}$, the interior conditions are one member of each of the five products

$$
F_{\alpha}^{(0)} u_{\alpha}^{(0)}, \quad F_{\beta}^{(0)} u_{\beta}^{(0)}, \quad F_{2}^{(0)} u_{2}^{(0)}, \quad F_{\alpha}^{(1)} u_{\alpha}^{(1)}, \quad F_{\beta}^{(1)} u_{\beta}^{(1)} .
$$

In terms of orthogonal coordinates $n, s, x_{2}$, the edge conditions are one member of each of the five products

$$
T_{n n}^{(0)} u_{n}^{(0)}, \quad T_{n s}^{(0)} u_{s}^{(0)}, \quad T_{n 2}^{(0)} u_{2}^{(0)}, \quad T_{n n}^{(1)} u_{n}^{(1)}, \quad T_{n s}^{(1)} u_{s}^{(1)} .
$$

The first two of these products give the boundary conditions of generalized plane stress and the last three give the boundary conditions of Reissner's equations of flexure [11].

8. Orthogonal functions. A theorem analogous to Clebsch's* may also be established. Consider two solutions

$$
\begin{aligned}
& \left(u_{j}^{(0)}, u_{b}^{(1)}\right)=\left(u_{j}^{(0) r}, u_{b}^{(1) r}\right) \exp i \omega_{r} t \\
& \left(u_{i}^{(0)}, u_{b}^{(1)}\right)=\left(u_{j}^{(0) s}, u_{b}^{(1) s}\right) \exp i \omega_{s} t
\end{aligned}
$$

of the homogeneous $\left(F_{i}^{(0)}=F_{b}^{(1)}=0\right)$ stress-equations of motion, so that the equations

$$
\begin{aligned}
& 2 \rho h \omega_{r}^{2} u_{i}^{(0) r}=-T_{i j, i}^{(0) r}, \\
& \frac{2}{3} \rho h^{3} \omega_{r}^{2} u_{b}^{(1) r}=-T_{a b}^{(1) r}+T_{2 b}^{(0) r}, \\
& -2 \rho h \omega_{s}^{2} u_{i}^{(0) s}=T_{i j, i}^{(0) s}, \\
& -\frac{2}{3} \rho h^{3} \omega_{s}^{2} u_{b}^{(1) s}=T_{a b}^{(1) s}-T_{2 b}^{(0) s}
\end{aligned}
$$

are satisfied. Multiplying these equations by $u_{i}^{(0) s}, u_{b}^{(1) s}, u_{i}^{(0) r}, u_{b}^{(1) r}$, respectively, adding and integrating over the area of the plate, we obtain, on the left hand side,

$$
2 h \rho\left(\omega_{r}^{2}-\omega_{s}^{2}\right) \int_{A}\left(u_{j}^{(0) r} u_{j}^{(0) s}+\frac{1}{3} h^{2} u_{b}^{(1) r} u_{b}^{(1) s}\right) d A
$$

and, on the right hand side,

$$
\int_{A}\left(T_{i j, i}^{(0) s} u_{j}^{(0) r}-T_{i j, i}^{(0) r} u_{j}^{(0) s}+T_{a b, a}^{(1) s} u_{b}^{(1) r}-T_{a b, a}^{(1) r} u_{b}^{(1) s}+T_{2 b}^{(0) r} u_{b}^{(1) s}-T_{2 b}^{(0) s} u_{b}^{(1) r}\right) d A .
$$

The latter, by a process similar to that employed in the preceding section, may be transformed to

$$
\begin{aligned}
2 h \int_{A}\left[g _ { i j k l } ^ { * } \left(S_{i j}^{(0) s} S_{k l}^{(0) r}-S_{i j}^{(0) r}\right.\right. & \left.\left.S_{k l}^{(0) s}\right)+\frac{1}{3} h^{2} \gamma_{a b c d}\left(S_{a b}^{(1) s} S_{c d}^{(1) r}-S_{a b}^{(1) r} S_{c d}^{(1) s}\right)\right] d A \\
& \quad \oint_{c} n_{a}\left(T_{a j}^{(0) s} u_{j}^{(0) r}-T_{a j}^{(0) r} u_{j}^{(0) s}+T_{a b}^{(1) s} u_{b}^{(1) r}-T_{a b}^{(1) r} u_{b}^{(1) s}\right) d s .
\end{aligned}
$$

The integrand in the surface integral vanishes identically. The integrand in the line

${ }^{*}$ Reference [6], p. 180. 
integral vanishes for any one of the thirty-two sets of edge-conditions obtained by equating to zero one member of each of the five products in (38), or for any of the associated conditions of elastic support. Then, if $w_{r} \neq w_{s}$

$$
\int_{A}\left(u_{j}^{(0) r} u_{j}^{(0) s}+\frac{1}{3} h^{2} u_{b}^{(1) r} u_{b}^{(1) s}\right) d A=0 .
$$

Some observations may be made regarding normal modes of rectangular plates. In the general triclinic case there are no solutions of the displacement-equations of motion, (22)-(23), with each of the five components of displacement a single product-function. However, such solutions are possible for monoclinic and higher symmetries.

Thus, in the monoclinic case, where (33) hold, (22) and (23) are satisfied by the four sets of displacements (and no others)

$$
\begin{gathered}
u_{1}^{(0)}=A_{1}^{(0)} \cos \left(\xi x_{1}+p \pi / 2\right) \sin \left(\zeta x_{3}+q \pi / 2\right) \exp i \omega t, \\
u_{2}^{(0)}=A_{2}^{(0)} \sin \left(\xi x_{1}+p \pi / 2\right) \cos \left(\zeta x_{3}+q \pi / 2\right) \exp i \omega t, \\
u_{3}^{(0)}=A_{3}^{(0)} \sin \left(\xi x_{1}+p \pi / 2\right) \cos \left(\zeta x_{3}+q \pi / 2\right) \exp i \omega t, \\
u_{1}^{(1)}=A_{1}^{(1)} \cos \left(\xi x_{1}+p \pi / 2\right) \cos \left(\zeta x_{3}+q \pi / 2\right) \exp i \omega t, \\
u_{3}^{(1)}=A_{3}^{(1)} \sin \left(\xi x_{1}+p \pi / 2\right) \sin \left(\zeta x_{3}+q \pi / 2\right) \exp i \omega t, \\
p=0,1 ; \quad q=0,1
\end{gathered}
$$

subject to a quintic equation relating $\omega^{2}, \xi^{2}$ and $\zeta^{2}$. For a plate $x_{1}= \pm h_{1}, x_{3}= \pm h_{3}$, normal modes, in which each displacement is a single product function, are possible if and only if, on $x_{1}= \pm h_{1}$,

$$
\begin{aligned}
u_{2}^{(0)} & =u_{3}^{(0)}=u_{3}^{(1)}=T_{11}^{(0)}=T_{11}^{(1)}=0 \\
\text { or } \quad T_{12}^{(0)} & =T_{13}^{(0)}=T_{13}^{(1)}=u_{1}^{(0)}=u_{1}^{(1)}=0
\end{aligned}
$$

and, on $x_{3}= \pm h_{3}$,

$$
\begin{aligned}
& u_{2}^{(0)}=u_{3}^{(0)}=u_{1}^{(1)}=T_{13}^{(0)}=T_{33}^{(1)}=0 \\
& \text { or } \quad T_{23}^{(0)}=T_{33}^{(0)}=T_{13}^{(1)}=u_{1}^{(0)}=u_{3}^{(1)} 0,
\end{aligned}
$$

whence

$$
\xi=m \pi / 2 h_{1}, \quad \zeta=n \pi / 2 h_{3} ; \quad m, n=0,1,2 \cdots
$$

and the frequencies are given by the five roots of the quintic for each $m$ and $n$.

The first set of (40) and the first set of (41) are the conditions analogous to simply supported edges in the elementary theory of flexure; but note that, in the present case, (41) are not obtained from (40) by interchange of indices 1 and 3. As in the elementary theory, solutions in closed form are not possible, in general, if all four edges are free; but closed solutions may be obtained with one pair of parallel edges free and the other pair under conditions (40) or (41). Then each of the displacements in (39) is given by a sum of five functions and one of the sets of roots (42) is replaced by the roots of a $5 \times 5$ transcendental, determinantal equation. There is one exceptional case of a solution in closed form, with all four edges free [19], analogous to the Lamé modes of an isotropic plate. 


\section{REFERENCES}

1. A. L. Cauchy, Sur l'équilibre et le mouvement d'une plaque élastique dont l'élasticité n'est pas la même dans tous les sens, Exercices de Mathématique 4, 1-14 (1829)

2. R. D. Mindlin, Thickness-shear and flexural vibrations of crystal plates, J. Appl. Phys. 22, 316-323 (1951)

3. S. D. Poisson, Mémoire sur l'équilibre et le mouvement des corps elastiques, Mém. Acad. Sci., Paris, Ser. 2, 8 357-570 (1829)

4. G. Kirchhoff, Über das Gleichgewicht und die Bewegung einer elastichen Scheibe, Crelles J. 40, 51-88 (1850)

5. H. Ekstein, High frequency vibrations of thin crystal plates, Phys. Rev. 68, 11-23 (1945)

6. A. E. H. Love, A treatise on the mathematical theory of elasticity, Cambridge University Press, London, 1927 , p. 106

7. R. D. Mindlin, An introduction to the mathematical theory of vibrations of elastic plates, U. S. Army Signal Corps, Fort Monmouth, N. J., Contract DA-36-039 SC-56772, pp. 6.02-6.04 (1955)

8. W. Voigt, Lehrbuch der Krystallphysik, B. G. Teubner, Leipzig, 2nd ed., 1928, p. 698

9. R. D. Mindlin, Influence of rotatory inertia and shear on flexural vibrations of isotropic, elastic plates, J. Appl. Mech. 18, 31-38 (1951)

10. S. Timoshenko, On the correction for shear of the differential equation for transverse vibrations of prismatic bars, Phil. Mag., Ser. 6, 41, 744-746 (1921)

11. E. Reissner, On bending of elastic plates, Quart Appl. Math. 5, 55-68 (1947)

12. I. Koga, Thickness vibrations of piezoelectric oscillating crystals, Physics 3, 70-80 (1932)

13. R. D. Mindlin, Waves and vibrations in isotropic, elastic plates, Proc. 1st Symp. Naval Structural Mechanics, Pergamon Press, New York, 1960, pp. 199-232

14. W. P. Mason, Piezoelectric crystals and their application to ultrasonics, D. Van Nostrand Co., New York, 1950, Chap. VI

15. R. Bechmann, Elastic and piezoelectric constants of aplha-quartz, Phys. Rev. 110, 1060-1061 (1958)

16. E. G. Newman and R. D. Mindlin, Vibrations of a monoclinic crystal plate, J. Acoust. Soc. Am. 29 1206-1218 (1957)

17. R. K. Kaul and R. D. Mindlin, Vibrations of a monoclinic crystal plate, II, (forthcoming)

18. D. C. Gazis and R. D. Mindlin, Extensional vibrations and waves in a circular disk and a semi-infinite plate, J. Appl. Mech. 27, (1960)

19. R. D. Mindlin, Simple modes of vibration of crystals, J. Appl. Phys. 27, 1462-1466 (1956) 